\title{
Skin Immunosenescence and Type 2 Inflammation: A Mini-Review With an Inflammaging Perspective
}

\author{
Bangtao Chen ${ }^{1}$, Jing Yang ${ }^{1}$, Yao Song ${ }^{2}$, Daojun Zhang ${ }^{2}$ and Fei Hao ${ }^{2 *}$ \\ ${ }^{1}$ Department of Dermatology, Chongqing University Three Gorges Hospital, School of Medicine, Chongqing University, \\ Chongqing, China, ${ }^{2}$ Department of Dermatology, The Third Affiliated Hospital of Chongqing Medical University, Chongqing, China
}

Skin-resident stromal cells, including keratinocytes, fibroblasts, adipocytes, and immune cells including Langerhans cells, dendritic cells, T cells, and innate lymphoid cells, and their functional products work in concert to ensure the realization of skin barrier immunity. However, aging-induced immunosenescence predisposes the elderly to pruritic dermatoses, including type 2 inflammation-mediated. Inflammaging, characterized by chronic low level of pro-inflammatory cytokines released from senescent cells with the senescence-associated secretory phenotype (SASP), may drive immunosenescence and tangle with type 2 inflammatory dermatoses. The present mini-review summarizes current

OPEN ACCESS

Edited by:

Mingxing Lei,

Chongqing University, China

Reviewed by:

Ling-juan Zhang,

Xiamen University, China

Zhirong Yao,

Shanghai Jiao Tong University, China

*Correspondence:

Fei HaO

haofei62@medmail.com.cn

Specialty section:

This article was submitted to

Cell Death and Survival,

a section of the journal

Frontiers in Cell and Developmental

Biology

Received: 14 December 2021

Accepted: 17 January 2022

Published: 24 February 2022

Citation:

Chen B, Yang J, Song Y, Zhang D and Hao $F$ (2022) Skin Immunosenescence and Type 2 Inflammation: A Mini-

Review With an

Inflammaging Perspective.

Front. Cell Dev. Biol. 10:835675

doi: $10.3389 /$ fcell.2022.835675 evidence on immunosenescence and type 2 inflammation in the skin and further focuses on future needs from an inflammaging perspective to clarify their complexity.

Keywords: immunosenescence, inflammaging, skin aging, dermatosis, mini-review and challenges

\section{INTRODUCTION}

The skin is the largest active immune organ, covering the body's outermost layer and performing the function of resisting external stimulus, thus maintaining skin homeostasis. Skin barrier inevitably undergoes characteristically immunological declines with advancing age, termed skin immunosenescence. Higher incidences of many dermatoses such as infectious diseases, noncommunicable autoimmune diseases, and cutaneous malignancies, and more pathological states such as unspecific itchiness and delayed wound healing are observed in the elderly alongside immunosenescence (Farage et al., 2009). Senescent cells remain senescence-associated secretory phenotype (SASP) secreting low-level pro-inflammatory cytokines including CRP, IL-1 $\beta$, IL-6, and TNF- $\alpha$, which is usually referred to inflammaging (Lopes-Paciencia et al., 2019; Fitsiou, et al., 2021). Type 2 inflammatory dermatosis such as atopic dermatitis (AD), chronic spontaneous urticaria (CSU), and bullous pemphigoid (BP) frequently affect the elderly and are presumed to be correlated with skin immunosenescence. Moreover, the diseases affecting the elderly are prone to more severity, therapeutic resistance, and longer duration. The current mini-review focuses on skin immunosenescence and type 2 inflammation and present future needs from an inflammaging perspective, promising better management of type 2 inflammatory dermatosis in the elderly (Figure 1).

\section{SKIN BARRIER IMMUNITY}

Skin-resident stromal cells, including keratinocytes, fibroblasts, and adipocytes, and immune cells including Langerhans cells (LCs), dendritic cells (DCs), T cells, and innate lymphoid cells (ILCs) 


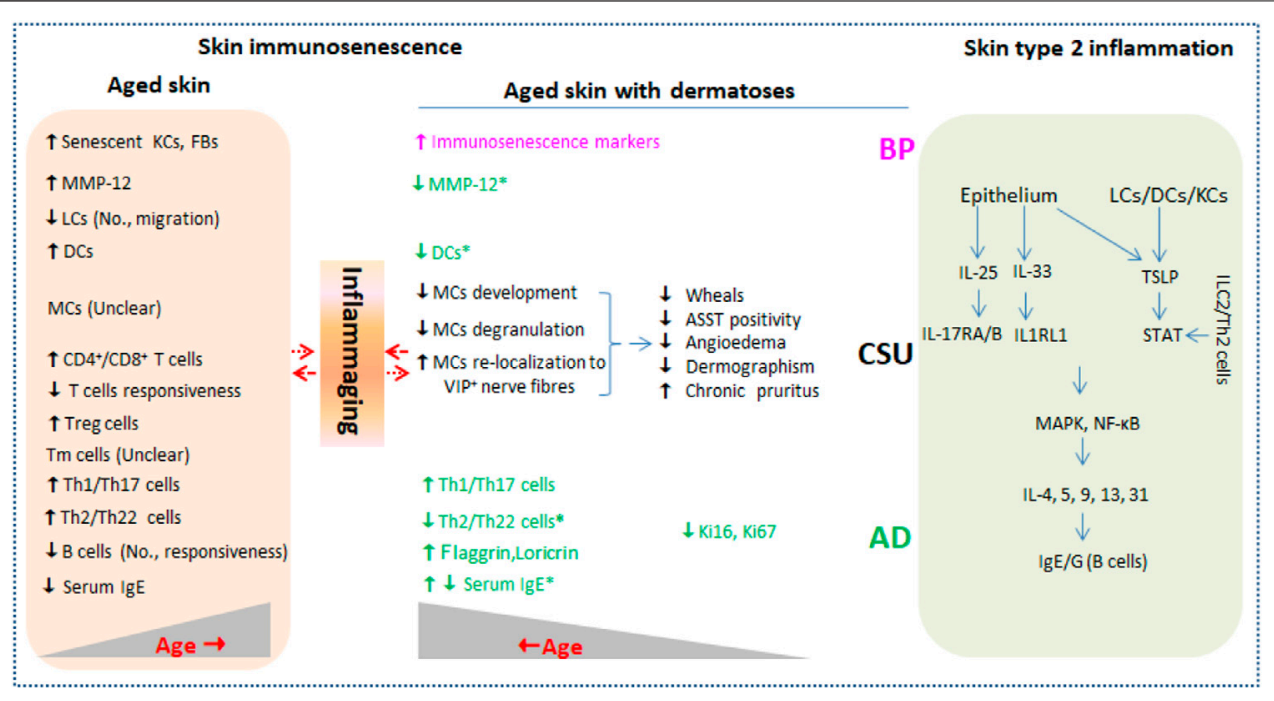

FIGURE 1 | Current evidence of immunosenescence in aged skin with or without type 2 inflammation dermatoses. Type 2 inflammation dermatoses such as AD, CSU, and BP are driven by key cytokines including IL-25, IL-33, and TSLP released from damaged epithelium and LCs/DCs (right column). Skin barrier inevitably undergoes characteristically immunological changes (skin immunosenescence) during aging in healthy individuals (left column) and in individuals affected by type 2 inflammation dermatoses. Inflammaging that is characterized by low level of pro-inflammatory cytokines including CRP, IL-1 $\beta$, IL-6, and TNF- $\alpha$ produced by senescent skin cells may be in complex interaction with the two conditions. No., number; KCs, keratinocytes; FBs, fibroblasts; LCs, Langerhans cells; DCs, dendritic cells; MCs, mast cells; ILC2, innate lymphoid cell 2; Tm, memory T cells; Ig, immune globulin; MMP-12, matrix metalloproteinase 12; ASST, autologous serum skin test; TSLP, thymic stromal lymphopoietin; STAT, signal transducer and activator of transcription; IL1RL1, IL-1 receptor-like 1; AD, atopic dermatitis; CSU, chronic spontaneous urticaria; BP, bullous pemphigoid.

together with their functional products ensure the realization of skin barrier immunity. The cells mentioned above work synergistically or antagonistically upon harmful environmental exposures challenge, leading to reinforced or compromised networks protecting the skin against damage or causing dermatosis, respectively.

\section{Stromal Cells}

Epidermal keratinocytes express and secrete antimicrobial ribonuclease RNase 7 and antimicrobial peptides adrenomedullin, $\beta$-defensins, and cathelicidin upon recognizing pathogenic components via its constitutive expressions of toll-like receptors (TLRs) on the cellular surface (Miller and Modlin, 2007; Köllisch, et al., 2005). In addition, keratinocytes function in the presentation of antigen from $\mathrm{CD} 4^{+}$ to $\mathrm{CD}^{+} \mathrm{T}$ cells and promote tissue repair via chemokine (IL-1 $\beta$, IL-8, and CCL20)-mediated leukocyte recruitment during early wound healing (Black, et al., 2007; Li, et al., 2017; Sperling, et al., 2012). They also serve as key sites for UVB-catalyzed production of active vitamin D3 (Zbytek, et al., 2008). Dermal fibroblasts are not only key to supporting wound healing through the secretion and remodeling of extracellular matrix (ECM) but also essential for facilitating innate immune response to microbial infections by secreting cytokines and chemokines with involvement of toll-like receptor activation (Ghetti, et al., 2018; Cole, et al., 2018; Haniffa, et al., 2007; Bautista-Hernández, et al., 2017). Interestingly, fibroblasts were uncovered to inhibit $\mathrm{T}$-cell proliferation and induce the production of immunoregulatory cytokines such as IL10 (Haniffa, et al., 2007). Moreover, adipocytes differentiated from dermal fibroblasts upon Staphylococcus aureus infection can also produce the antimicrobial peptide cathelicidin (Zhang, et al., 2015).

\section{Immune Cells}

LCs, the mononuclear phagocyte within the epidermis, not only produce antimicrobial peptide hBD3 and initiate a local immune response mainly by presenting antigens to $\mathrm{T}$ cells (Ferris, et al., 2013; Atmatzidis, et al., 2017; Pilkington, et al., 2018a), but also migrate to skin regional lymph nodes for enhancing immune response to exogenous antigens and promoting tolerance to selfantigens (West and Bennett, 2018; Atmatzidis, et al., 2017).

DCs and macrophages, the mononuclear phagocyte located in the dermis, are also the sentinels of the innate immunity working similarly to epidermal LCs. Dermal DCs comprise $\mathrm{CD}_{1 \mathrm{c}^{+}}$or $\mathrm{CD}_{141}{ }^{+}$myeloid and plasmacytoid forms, while the latter is hardly observed in steady-state skin (Collin and Bigley, 2018). Compared with their blood counterparts, normal dermal DCs associated with T-cell proliferation displayed an activated phenotype with increased expression of co-stimulatory receptors (McLellan, et al., 1998). Dermal macrophages are specifically labeled with CD163, and the cells also contribute to wound and nerve healing by suppressing inflammation upon tissue injury (Kolter, et al., 2019).

The same as skin LC and DC, B cells found in healthy skin are integral for presenting antigen at low concentration to $\mathrm{T}$ cells (Geherin, et al., 2012). Moreover, skin B cells also modulate inflammation response by secreting pro- or anti-inflammatory mediators (Debes, and McGettigan, 2019).

Skin-resident $\mathrm{T}$ cells derived from $\mathrm{T}$ cells differentiated and matured in the thymus via migration through the lymphatic or 
circulatory system. Phenotypically, $80 \%-90 \%$ of the skin T-cell pool is memory $\mathrm{T}(\mathrm{Tm})$ cells, and the remaining is recirculating T cells (Nguyen, et al., 2019). Tm cells have stronger immune surveillance against reinvasions, and expressions of CD69 and CD103 on cell surface commonly characterize this type of T cells (Mackay, et al., 2013). The number of CD4 ${ }^{+}$Tm cells is three and six times that of $\mathrm{CD}^{+}$Tm cells in the epidermis and dermis, respectively (Watanabe, et al., 2015). With a memory skinresident phenotype inducing immune tolerance, Foxp $3^{+}$ regulatory $\mathrm{T}$ cells (Tregs) are in close proximity to hair follicles where skin commensal-metabolized short-chain fatty acid sodium butyrate or UVB light increases Foxp $3^{+}$ expression in non-Tregs or drive Foxp $3^{+}$Tregs proliferation (Schwarz, et al., 2017; Yamazaki, et al., 2014; Scharschmidt, et al., 2017). Overall, $\alpha \beta \mathrm{T}$ cells dominate in the skin as in circulation (Nielsen, et al., 2017).

Cutaneous ILCs located in the epidermis and dermis are newly identified immune cells whose function is not fully understood, but influxes of ILC2 in $\mathrm{AD}$ and $\mathrm{LC} 1 / 3$ in psoriatic plaques were demonstrated (Brüggen, et al., 2016; Akdis, et al., 2020). Neutrophils are seldom in the skin, while they can infiltrate the skin upon exposure to a harmful stimulus (Rijken, et al., 2005). In addition, allergens or inflammatory irritants can induce the release of histamine and inflammatory mediators from cutaneous mast cells (MCs), mediating wheals and itch onset (Otsuka, and Kabashima, 2015).

\section{SKIN IMMUNOSENESCENCE}

The skin goes roughly through stages of immaturity, maturation, and decline over lifespan as with all other organs. Although incredibly durable, aging still causes skin structure and function changes, termed skin aging. This process is usually exaggerated by extrinsic exposures such as UVR. Morphologic and related functional changes in chronologically or intrinsically aged skin were summarized in a review conducted by Zouboulis, and Makrantonaki, (2011). In particular, immunosenescence contributes to the increased susceptibility to skin disorders with malignancies, infections, and autoimmunity in the elderly. Skin immunosenescence refers to declines in function or number of all skin cells responsible for immune surveillance (Corsini, et al., 2009). Senescent cells, promoted by telomere shortening and genome instability, remain SASP secreting low level of pro-inflammatory cytokines including IL-1 $\beta$, IL-6, and CRP, thus altering the skin's microenvironment (LopesPaciencia, et al., 2019; Fitsiou, et al., 2021). Presumably, skin inflammaging characterized by chronic low-level inflammation is believed to be the main driver for remodeling the immunological response in senescent skin cells (Ghosh, and Capell, 2016).

\section{Stromal Cell Senescence}

Dermal senescent fibroblasts accumulated with age and displayed SASP rich, thus maintaining inflammaging phenotype (Wlaschek, et al., 2021). Such changes contribute to disruptions of collagen homeostasis, delayed wound healing, and increased likelihood of skin tumorigenesis; however, its antibacterial immunity loss caused by aging has been associated with impaired adipocyte differentiation (Zhang, et al., 2019; Wasko, and Horsley, 2019). Compared with fibroblasts, the impact of accumulated senescent keratinocytes in the epidermis on inflammaging or antibacterial immunity is limited due to its higher turnover rate (Pilkington, et al., 2021).

\section{Immune Cell Senescence}

In aged skin, decreased proliferation of in situ LC progenitors causes a reduced number of LCs, and LCs are also less able to migrate from the epidermis in response to harmful stimulus due to the declined availability of local IL- $1 \beta$, which collectively contributes to impaired skin barrier integrity and diminished antimicrobial and tumor cell defense (Pilkington, et al., 2018b). In addition, LC-mediated skin barrier perturbation may facilitate the onset of skin inflammaging by initiating cytokine release from cutaneous cells (Wittmann, et al., 2014).

To some extent, the state of thymus and T cells in circulation are implicated in many dermatoses, which also reflect the profiles of skin-resident $\mathrm{T}$ cells. In geriatric individuals, circulating $\mathrm{T}$ cells in total number remain unchanged, accompanied by reduction of naive $\mathrm{T}$ cells due to thymic involution and increase of Tm cells since the prolonged exposure to external substances over the lifespan (Thomas, et al., 2020). However, little is known regarding the changes in skin-resident Tm cells during aging. A higher ratio of $\mathrm{CD}^{+}$to $\mathrm{CD}^{+} \mathrm{T}$ cells was found in aged skin than in young skin, indicating a more severe pro-inflammatory response phenotype (Zuelgaray, et al., 2019). Cytokines during inflammaging can be Th2 pattern dominant with an increased incidence of allergic diseases and Th1 pattern dominant with a higher frequency of chronic infections and neoplastic diseases. It was reported that Tregs numbers and immunosuppressive receptor PD-1 increased in aged skin, thus causing reactivation of infectious diseases or skewing inflammatory microenvironment by suppressing both Th1 and Th2 responses (Lages, et al., 2008). Additionally, a diminished response of $\mathrm{T}$ cells to specific antigens in advanced age may collectively explain why the chronic low-level inflammation characterizes the state of inflammaging (Bektas, et al., 2017).

Aging-related changes in skin B cells are similar to skin T cells except that B cells from the elderly are less efficiently stimulated. Thus, antibody generation decreases, and immune response to vaccines and antigens is weakened (Pinti, et al., 2016).

\section{IMMUNOSENESCENCE AND TYPE 2 INFLAMMATORY DERMATOSIS}

Type 2 inflammation phenotypes in skin and circulation are usually in traffic with each other and remain consistent. IL-25, IL33, and thymic stromal lymphopoietin (TSLP) released from damaged epithelium directly activate the production of IL-4, 5, 9, 13, and 31 from ILC2 and Th2 cells, thus characterizing type 2 inflammation immunity (Akdis, et al., 2020). Both IL-25 and IL33 can activate MAPK and NK- $\kappa B$ signaling pathways via binding to IL-17RA/B and IL-1 receptor-like 1 (IL1RL1), respectively (Akdis, et al., 2020; Deng, et al., 2021). By activating signal 
transducer and activator of transcription (STAT), skin LC/DCderived and keratinocyte-derived TSLP are critical for Th2-type immune responses and mediating pruritus exacerbation, respectively (Kim et al., 2013). In addition, humoral immunity characterized by allergen-specific antibody IgE or autoantigenspecific autoantibody IgG matured by IL-13 and IL-4 is also involved in type 2 inflammatory dermatosis such as AD, CSU, and BP (Gandhi, et al., 2016).

\section{Atopic Dermatitis}

Globally, $10 \%$ of adults and $1 \%-3 \%$ of elderly populations are troubled by AD (Lloyd-Lavery, et al., 2019; Williamson, et al., 2020). Moreover, the increasing predisposition of late $\mathrm{AD}$ development in older adults is due to exposure-induced epidermal barrier malfunction and immunosenescence-caused chronic itch in advanced age. The core of $\mathrm{AD}$ is skin inflammation involving IgE produced by $\mathrm{B}$ cells and inflammatory mediators of T-cell origin, while Th2 cytokines dominate in the inflammation milieu (Tanei, and Hasegawa, 2016; Tanei, et al., 2013). Th2/Th22 cytokines in skin increase during aging in healthy individuals, while the opposite phenomenon is observed in older AD patients (Bocheva, et al., 2021; Gittler, et al., 2012). With age progressing, Th1- and Th17related mediators in lesioned and non-lesioned skin in individuals suffering from $\mathrm{AD}$ are markedly increased, as observed in healthy adults (Bocheva, et al., 2021; Gittler, et al., 2012). Zhou et al. showed that inflammatory DCs in the skin and cutaneous expression of matrix metalloproteinase 12 (MMP-12) were reduced in both affected and unaffected skin in $\mathrm{AD}$ with aging (Agrawal, et al., 2012; Zhou, et al., 2019). Reduction in specific and total serum IgE with aging in patients with allergic rhinitis, asthma, or insect allergy implies a decreasing proportion of extrinsic atopy among older adults; however, the association between serum $\mathrm{IgE}$ and aging in $\mathrm{AD}$ patients remains inconsistent (Zhou, et al., 2019; Mediaty, and Neuber, 2005). In addition, aging-related increment in terminal keratinocyte differentiation markers (filaggrin and loricrin) and decrement in epidermal hyperplasia markers (Ki16 and Ki67) were also observed in AD (Zhou, et al., 2019), which might be attributable to attenuation in the Th2/Th22 cytokine axes (Boniface, et al., 2005); moreover, it reveals a critical role for crosstalk between immune cell senescence and stromal cell-mediated immunity impairment in severity of geriatric $\mathrm{AD}$.

\section{Chronic Spontaneous Urticaria}

Traditionally, CSU is an allergic dermatosis mediated by degranulation and histamine released from skin MCs or basophils (Bracken, et al., 2019). As mounting CSU patients show antihistamine resistance, it is supposed to be $\mathrm{T}$ cellmediated with emerging evidence that concentrations of circulating cytokines released from Th1/Th2 and Th17 cells correlated positively with disease severity in our previous study (Chen, et al., 2018). Kay et al. added the finding that increased expressions of IL-25, IL-33, and TSLP in skin wheals of patients with CSU further accurately characterize the pathogenesis and categorize it as type 2 inflammatory dermatosis (Kay, et al., 2015; Vadasz, and Toubi, 2015). In retrospective investigations performed in localized areas, older CSU patients made up $9.4 \%-25 \%$ of the CSU population. Furthermore, fewer wheals, lower rates of ASST positivity, angioedema, and dermographism, and more comorbidities were reported in elderly patients with CSU diagnosis (Chen, et al., 2012; Magen, et al., 2013). The atypical symptoms are pertinent to aging-related immunosenescence. For one thing, stromal-cell functional impairment with aging was proved to cause a decline in MC development (Tsuboi et al., 2012). For another, skin MCs accumulated while their degranulation capability was reduced with aging. Furthermore, they relocalize to the papillary dermis, where MCs keep in closer proximity to macrophages and $\mathrm{VIP}^{+}$nerve fibers while the association with dermal vasculature is weakened (Pilkington, et al., 2019). Unfortunately, little is known regarding alterations of number, function, and crosstalk among MCs, basophils, and $\mathrm{T}$ and $\mathrm{B}$ cells in elderly individuals with CSU.

\section{Bullous Pemphigoid}

$\mathrm{BP}$, an autoimmune blistering dermatosis in the elderly mediated by IgG autoantibodies to skin hemidesmosome proteins (BP180 and/or BP230) and activation of complement component C3, is characterized by urticarial plaques, tense blisters, and intractable pruritus (Bağc1, et al., 2017). In BP development, autoreactive $\mathrm{T}$ cells work cooperatively. Increased circulating Th2 cells and IL4 promote $\mathrm{B}$-cell proliferation, antibody production, and immunoglobulin class-switching, while skin-resident Th17 cells and IL-17 activate local neutrophil-mediated inflammatory response, thus causing tissue damage (Fang, et al., 2020; Boehncke, and Brembilla, 2019). In recent studies, specific anti-BP180/230 IgE in BP were detected by immunoassays; furthermore, positive associations between $\operatorname{IgE}$ content and Th2 cell-specific cytokines IL-4/-13 and symptomatic disease phenotypes were shown (Cozzani, et al., 2018; Messingham, et al., 2019). The finding that IgE-driven BP promises the therapeutic regimes using Th2 inhibitors in BP-affected frail patients with good safety and ideal effectiveness. It was reported that disease clearance or satisfactory response was achieved in 12 of $13 \mathrm{BP}$ patients (an average age of 76.8 years) treated with Dupilumab, an IL-4 receptor alpha antagonist with the property of inhibiting IL-4/-13 signaling and IgE secretion (Abdat, et al., 2020). Immunosenescence-related aging is conceivably responsible for the increased incidence of $\mathrm{BP}$ in the elderly (Pietkiewicz, et al., 2016; Yaar, and Gilchrest, 1987). However, fewer studies focus on the effect of immunosenescence or inflammaging on pathophysiological characteristics of $\mathrm{BP}$, and only a meeting paper uncovered increased markers of immunosenescence in BP patients (Noe, et al., 2015).

\section{FUTURE NEEDS}

Too many questions regarding skin immunosenescence and type 2 inflammations need to be answered.

Firstly, existing studies fail to provide direct and strong evidence for the involvement of immunosenescence in type 2 
inflammatory dermatosis. Distinctive clinical features and incidences of type 2 inflammatory dermatosis between the young and the elderly are observed, and the difference is often thought to be caused by aging-related changes including immunosenescence, but direct evidence remains insufficient. As evidenced by the recent discovery of TH2-interacting fascial fibroblasts (TIFFs) in mouse and human skin, skinresident or -infiltrating immune cells and stromal cells are complexly interacting and influence each other throughout life (Boothby, et al., 2021). They undergo structural and functional alterations simultaneously, but overall, inflammaging phenotype characterizes the skin microenvironment during normal aging. In aged individuals with type 2 inflammatory dermatosis, the relationship of the skin microenvironment with inflammaging and changes to cutaneous immunity is more complex due to repeated scratching caused by uncontrolled itchiness. Therefore, it is far-fetched to conclude that a specific pathophysiological change is independently caused by a specific senescent cell alone or the disease itself. In particular, further exploration of the associations between remolding of senescent fibroblast-released ECM and type 2 inflammation in aged skin will provide new insights into strategies used in related dermatosis.

Secondly, could skin immunosenescence contribute to systemic immunosenescence or vice versa from the perspective of inflammaging? A shining shared feature of type 2 inflammatory dermatoses in the young or elderly is the presence of Th2 cytokines in circulation and the lesions, and inflammation state in lesions is proposed to be orchestrated by systemic inflammation phenotype (Rafei-Shamsabadi, et al., 2019; Pezzolo and Naldi, 2020). Given this, the reverse argument is worth further considering, especially in the elderly with impaired skin barrier as epidermal abnormality in $\mathrm{AD}$ has been proposed to drive systemic inflammation (Elias and Steinhoff, 2008). Hu et al. showed that tape stripping-induced epidermal dysfunction led to an age-associated increase in levels of circulating inflammatory cytokines in mice (Hu, et al., 2017), and Ye et al. also provided the evidence that correction of epidermal function by emollient lowered systemic inflammaging measured by circulating levels of IL- 6 and TNF- $\alpha$ in chronically aged human (Ye, et al., 2019). These studies may collectively support the thesis that epidermal dysfunction-mediated immunosenescence could contribute to the onset or severity of type 2 inflammatory dermatosis with systemic inflammation involved in the elderly. However, more investigations are warranted to confirm the thesis by untangling their cause and effect.

On the contrary, the complexity of systemic and tissue inflammaging can also be witnessed in the efficacy of antiinflammaging agents. Anti-inflammaging drugs indeed hold promise for increasing healthy aging, and much effort aimed at slowing aging by targeting inflammaging has been conducted (Partridge, et al., 2020; Suggs, et al., 2014). Rapamycin, metformin, and various botanicals showed delaying the aging process by inhibiting cellular senescence dependent or independent of their anti-inflammaging properties (Partridge, et al., 2020; Suggs, et al., 2014). For example, topical rapamycin, an FDA-approved agent, showed no beneficial effects in inflammaging (Correia-Melo, et al., 2019) but improved histological appearance of aged skin by reducing fibroblast senescence and increasing collagen VII (Chung, et al., 2019; Qin, et al., 2018). As such, the correction of the impaired skin barrier by anti-inflammaging agents preventing or mitigating systemic inflammaging is meaningful and easily articulable.

Thirdly, other intrinsic drivers of skin immunosenescence or inflammaging should also be identified in terms of the organism as a whole. Changes to gut and skin microbiota, mitochondrial damage-associated molecular patterns (DAMPs), abnormal activity of coagulation and fibrinolysis, complements, and vitamin D3 deficiency during aging have also been linked with type 2 inflammation dermatoses (Nakahara, et al., 2021; SánchezBorges et al., 2018; Hashimoto, et al., 2020). Whether the correction of abnormalities benefits improvement of related dermatoses through immunosenescence retardation remains to be further investigated.

Lastly, skin immunosenescence can be partly determined with flow cytometry and immunohistochemistry by frequency assessments of senescent immune cells due to their end-stage differentiated and cell-specific markers; however, no techniques are available for assessing inflammaging caused by indicated senescent cells in vivo. It appears that all immune- or nonimmune-senescent cells possess SASP properties releasing low levels of IL-1 $\beta$, IL- 6 , TNF- $\alpha$, and CRP. These pro-inflammation mediators that can shuttle through skin and circulation are nonspecific for SASP-centered inflammaging. More than that, they can be transiently modulated by acute or persistently modulated by chronic inflammatory diseases, including type 2 inflammation dermatoses in young or older populations. Meanwhile, immunosenescence and inflammaging in the skin are mutually regulated, but they do not always parallel, especially for senescent cells in the end stage. Herein, screening of reasonable indicators for inflammaging in the elderly with and/or without inflammation dermatosis via longitudinal data from large samples is expected.

\section{AUTHOR CONTRIBUTIONS}

FH designed the study; BC and YS wrote the manuscript; DZ and JY revised the manuscript.

\section{FUNDING}

This work was funded by grants from the National Natural Science Foundation of China (82003337), the China Postdoctoral Science Foundation (2020M683268), the Chongqing Natural Science Foundation (cstc2020jcyj-bshX0023), the Postdoctoral Foundation of Chongqing Medical University (R9001), and Funding for Key Disciplines of Third Affiliated Hospital of Chongqing Medical University (ZK201902).

\section{ACKNOWLEDGMENTS}

We thank Professor Alan C. Zheng for his help in language editing. 


\section{REFERENCES}

Abdat, R., Waldman, R. A., de Bedout, V., Czernik, A., Mcleod, M., King, B., et al. (2020). Dupilumab as a Novel Therapy for Bullous Pemphigoid: A Multicenter Case Series. J. Am. Acad. Dermatol. 83, 46-52. doi:10.1016/j.jaad.2020.01.089

Agrawal, A., Sridharan, A., Prakash, S., and Agrawal, H. (2012). Dendritic Cells and Aging: Consequences for Autoimmunity. Expert Rev. Clin. Immunol. 8, 73-80. doi:10.1586/eci.11.77

Akdis, C. A., Arkwright, P. D., Brüggen, M.-C., Busse, W., Gadina, M., GuttmanYassky, E., et al. (2020). Type 2 Immunity in the Skin and Lungs. Allergy 75, 1582-1605. doi:10.1111/all.14318

Atmatzidis, D. H., Lambert, W. C., and Lambert, M. W. (2017). Langerhans Cell: Exciting Developments in Health and Disease. J. Eur. Acad. Dermatol. Venereol. 31, 1817-1824. doi:10.1111/jdv.14522

Bağcl, I. S., Horváth, O. N., Ruzicka, T., and Sárdy, M. (2017). Bullous Pemphigoid. Autoimmun. Rev. 16, 445-455. doi:10.1016/j.autrev.2017.03.010

Bautista-Hernández, L. A., Gómez-Olivares, J. L., Buentello-Volante, B., and Bautista-de Lucio, V. M. (2017). Fibroblasts: The Unknown Sentinels Eliciting Immune Responses against Microorganisms. Eur. J. Microbiol. Immunol. 7, 151-157. doi:10.1556/1886.2017.00009

Bektas, A., Schurman, S. H., Sen, R., and Ferrucci, L. (2017). Human T Cell Immunosenescence and Inflammation in Aging. J. Leukoc. Biol. 102, 977-988. doi:10.1189/jlb.3RI0716-335R

Black, A. P. B., Ardern-Jones, M. R., Kasprowicz, V., Bowness, P., Jones, L., Bailey, A. S., et al. (2007). Human Keratinocyte Induction of Rapid Effector Function in Antigen-specific Memory CD4+ and CD8+ T Cells. Eur. J. Immunol. 37, 1485-1493. doi:10.1002/eji.200636915

Bocheva, G. S., Slominski, R. M., and Slominski, A. T. (2021). Immunological Aspects of Skin Aging in Atopic Dermatitis. Int. J. Mol. Sci. 22, 5729. doi:10. 3390/ijms22115729

Boehncke, W.-H., and Brembilla, N. C. (2019). Autoreactive T-Lymphocytes in Inflammatory Skin Diseases. Front. Immunol. 10, 1198. doi:10.3389/fimmu. 2019.01198

Boniface, K., Bernard, F.-X., Garcia, M., Gurney, A. L., Lecron, J.-C., and Morel, F. (2005). IL-22 Inhibits Epidermal Differentiation and Induces Proinflammatory Gene Expression and Migration of Human Keratinocytes. J. Immunol. 174, 3695-3702. doi:10.4049/jimmunol.174.6.3695

Boothby, I. C., Kinet, M. J., Boda, D. P., Kwan, E. Y., Clancy, S., Cohen, J. N., et al. (2021). Early-life Inflammation Primes a T Helper 2 Cell-Fibroblast Niche in Skin. Nature 599, 667-672. doi:10.1038/s41586-021-04044-7

Bracken, S. J., Abraham, S., and MacLeod, A. S. (2019). Autoimmune Theories of Chronic Spontaneous Urticaria. Front. Immunol. 10, 627. doi:10.3389/fimmu. 2019.00627

Brüggen, M.-C., Bauer, W. M., Reininger, B., Clim, E., Captarencu, C., Steiner, G. E., et al. (2016). In Situ Mapping of Innate Lymphoid Cells in Human Skin: Evidence for Remarkable Differences between Normal and Inflamed Skin. J. Invest. Dermatol. 136, 2396-2405. doi:10.1016/j.jid.2016.07.017

Chen, Q., Zhong, H., Chen, W. C., Zhai, Z., Zhou, Z., Song, Z., et al. (2018). Different Expression Patterns of Plasma Th1-, Th2-, Th17- and Th22-Related Cytokines Correlate with Serum Autoreactivity and Allergen Sensitivity in Chronic Spontaneous Urticaria. J. Eur. Acad. Dermatol. Venereol. 32, 441-448. doi:10.1111/jdv.14541

Chen, Y.-J., Wu, C. Y., Shen, J. L., Chen, T. T., and Chang, Y. T. (2012). Cancer Risk in Patients with Chronic Urticaria. Arch. Dermatol. 148, 103-108. doi:10.1001/ archdermatol.2011.682

Chung, C. L., Lawrence, I., Hoffman, M., Elgindi, D., Nadhan, K., Potnis, M., et al. (2019). Topical Rapamycin Reduces Markers of Senescence and Aging in Human Skin: an Exploratory, Prospective, Randomized Trial. GeroScience 41, 861-869. doi:10.1007/s11357-019-00113-y

Cole, M. A., Quan, T., Voorhees, J. J., and Fisher, G. J. (2018). Extracellular Matrix Regulation of Fibroblast Function: Redefining Our Perspective on Skin Aging. J. Cel Commun. Signal. 12, 35-43. doi:10.1007/s12079-018-0459-1

Collin, M., and Bigley, V. (2018). Human Dendritic Cell Subsets: an Update. Immunology 154, 3-20. doi:10.1111/imm.12888

Correia-Melo, C., Birch, J., Fielder, E., Rahmatika, D., Taylor, J., Chapman, J., et al. (2019). Rapamycin Improves Healthspan but Not Inflammaging in Nfkb1 -/Mice. Aging cell 18, e12882. doi:10.1111/acel.12882
Corsini, E., Racchi, M., Lucchi, L., Donetti, E., Bedoni, M., Viviani, B., et al. (2009). Skin Immunosenescence: Decreased Receptor for Activated C Kinase-1 Expression Correlates with Defective Tumour Necrosis Factor- $\alpha$ Production in Epidermal Cells. Br. J. Dermatol. 160, 16-25. doi:10.1111/j.1365-2133.2008. 08885.x

Cozzani, E., Gasparini, G., Di Zenzo, G., and Parodi, A. (2018). Immunoglobulin E and Bullous Pemphigoid. Eur. J. Dermatol. 28, 440-448. doi:10.1684/ejd.2018. 3366

Debes, G. F., and McGettigan, S. E. (2019). Skin-Associated B Cells in Health and Inflammation. J. Immunol. 202, 1659-1666. doi:10.4049/jimmunol.1801211

Deng, C., Peng, N., Tang, Y., Yu, N., Wang, C., Cai, X., et al. (2021). Roles of IL-25 in Type 2 Inflammation and Autoimmune Pathogenesis. Front. Immunol. 12, 691559. doi:10.3389/fimmu.2021.691559

Elias, P. M., and Steinhoff, M. (2008). "Outside-to-Inside" (And Now Back to "Outside") Pathogenic Mechanisms in Atopic Dermatitis. J. Invest. Dermatol. 128, 1067-1070. doi:10.1038/jid.2008.88

Fang, H., Li, Q., and Wang, G. (2020). The Role of T Cells in Pemphigus Vulgaris and Bullous Pemphigoid. Autoimmun. Rev. 19, 102661. doi:10.1016/j.autrev. 2020.102661

Farage, M. A., Miller, K. W., Berardesca, E., and Maibach, H. I. (2009). Clinical Implications of Aging Skin. Am. J. Clin. Dermatol. 10, 73-86. doi:10.2165/ 00128071-200910020-00001

Ferris, L. K., Mburu, Y. K., Mathers, A. R., Fluharty, E. R., Larregina, A. T., Ferris, R. L., et al. (2013). Human Beta-Defensin 3 Induces Maturation of Human Langerhans Cell-like Dendritic Cells: an Antimicrobial Peptide that Functions as an Endogenous Adjuvant. J. Invest. Dermatol. 133, 460-468. doi:10.1038/jid.2012.319

Fitsiou, E., Pulido, T., Campisi, J., Alimirah, F., and Demaria, M. (2021). Cellular Senescence and the Senescence-Associated Secretory Phenotype as Drivers of Skin Photoaging. J. Invest. Dermatol. 141 (4S), 1119-1126. doi:10.1016/j.jid. 2020.09.031

Gandhi, N. A., Bennett, B. L., Graham, N. M. H., Pirozzi, G., Stahl, N., and Yancopoulos, G. D. (2016). Targeting Key Proximal Drivers of Type 2 Inflammation in Disease. Nat. Rev. Drug Discov. 15, 35-50. doi:10.1038/ nrd4624

Geherin, S. A., Fintushel, S. R., Lee, M. H., Wilson, R. P., Patel, R. T., Alt, C., et al. (2012). The Skin, a Novel Niche for Recirculating B Cells. J. Immunol. 188, 6027-6035. doi:10.4049/jimmunol.1102639

Ghetti, M., Topouzi, H., Theocharidis, G., Papa, V., Williams, G., Bondioli, E., et al. (2018). Subpopulations of Dermal Skin Fibroblasts Secrete Distinct Extracellular Matrix: Implications for Using Skin Substitutes in the Clinic. Br. J. Dermatol. 179, 381-393. doi:10.1111/bjd.16255

Ghosh, K., and Capell, B. C. (2016). The Senescence-Associated Secretory Phenotype: Critical Effector in Skin Cancer and Aging. J. Invest. Dermatol. 136, 2133-2139. doi:10.1016/j.jid.2016.06.621

Gittler, J. K., Shemer, A., Suárez-Fariñas, M., Fuentes-Duculan, J., Gulewicz, K. J., Wang, C. Q. F., et al. (2012). Progressive Activation of TH2/TH22 Cytokines and Selective Epidermal Proteins Characterizes Acute and Chronic Atopic Dermatitis. J. Allergy Clin. Immunol. 130, 1344-1354. doi:10.1016/j.jaci.2012. 07.012

Haniffa, M. A., Wang, X.-N., Holtick, U., Rae, M., Isaacs, J. D., Dickinson, A. M., et al. (2007). Adult Human Fibroblasts Are Potent Immunoregulatory Cells and Functionally Equivalent to Mesenchymal Stem Cells. J. Immunol. 179, 1595-1604. doi:10.4049/jimmunol.179.3.1595

Hashimoto, T., Kursewicz, C. D., Fayne, R. A., Nanda, S., Shah, S. M., Nattkemper, L., et al. (2020). Pathophysiologic Mechanisms of Itch in Bullous Pemphigoid. J. Am. Acad. Dermatol. 83, 53-62. doi:10.1016/j.jaad. 2019.07.060

Hu, L., Mauro, T. M., Dang, E., Man, G., Zhang, J., Lee, D., et al. (2017). Epidermal Dysfunction Leads to an Age-Associated Increase in Levels of Serum Inflammatory Cytokines. J. Invest. Dermatol. 137, 1277-1285. doi:10.1016/j. jid.2017.01.007

Kay, A. B., Clark, P., Maurer, M., and Ying, S. (2015). Elevations in T-Helper-2Initiating Cytokines (Interleukin-33, Interleukin-25 and Thymic Stromal Lymphopoietin) in Lesional Skin from Chronic Spontaneous ('idiopathic') Urticaria. Br. J. Dermatol. 172, 1294-1302. doi:10.1111/bjd.13621

Kim, B. S., Siracusa, M. C., Saenz, S. A., Noti, M., Monticelli, L. A., Sonnenberg, G. F., et al. (2013). TSLP Elicits IL-33-independent Innate Lymphoid Cell 
Responses to Promote Skin Inflammation. Sci. Transl. Med. 5, 170ra16. doi:10. 1126/scitranslmed.3005374

Köllisch, G., Kalali, B. N., Voelcker, V., Wallich, R., Behrendt, H., Ring, J., et al. (2005). Various Members of the Toll-like Receptor Family Contribute to the Innate Immune Response of Human Epidermal Keratinocytes. Immunology 114, 531-541. doi:10.1111/j.1365-2567.2005.02122.x

Kolter, J., Feuerstein, R., Zeis, P., Hagemeyer, N., Paterson, N., d'Errico, P., et al. (2019). A Subset of Skin Macrophages Contributes to the Surveillance and Regeneration of Local Nerves. Immunity 50, 1482-1497. e7. doi:10.1016/j. immuni.2019.05.009

Lages, C. S., Suffia, I., Velilla, P. A., Huang, B., Warshaw, G., Hildeman, D. A., et al. (2008). Functional Regulatory T Cells Accumulate in Aged Hosts and Promote Chronic Infectious Disease Reactivation. J. Immunol. 181, 1835-1848. doi:10. 4049/jimmunol.181.3.1835

Li, H., Li, H., Huo, R., Wu, P., Shen, Z., Xu, H., et al. (2017). Cyr61/CCN1 Induces CCL20 Production by Keratinocyte via Activating P38 and JNK/AP-1 Pathway in Psoriasis. J. Dermatol. Sci. 88, 46-56. doi:10.1016/j.jdermsci.2017.05.018

Lloyd-Lavery, A., Solman, L., Grindlay, D. J. C., Rogers, N. K., Thomas, K. S., and Harman, K. E. (20192016). What's New in Atopic Eczema? an Analysis of Systematic Reviews Published in 2016. Part 2: Epidemiology, Aetiology and Risk factorsPart 2: Epidemiology, Aetiology and Risk Factors. Clin. Exp. Dermatol. 44, 370-375. doi:10.1111/ced.13853

Lopes-Paciencia, S., Saint-Germain, E., Rowell, M.-C., Ruiz, A. F., Kalegari, P., and Ferbeyre, G. (2019). The Senescence-Associated Secretory Phenotype and its Regulation. Cytokine 117, 15-22. doi:10.1016/j.cyto.2019.01.013

Mackay, L. K., Rahimpour, A., Ma, J. Z., Collins, N., Stock, A. T., Hafon, M.L., et al. (2013). The Developmental Pathway for CD103+CD8+ TissueResident Memory T Cells of Skin. Nat. Immunol. 14, 1294-1301. doi:10. 1038/ni.2744

Magen, E., Mishal, J., and Schlesinger, M. (2013). Clinical and Laboratory Features of Chronic Idiopathic Urticaria in the Elderly. Int. J. Dermatol. 52, 1387-1391. doi:10.1111/ijd.12109

McLellan, A. D., Heiser, A., Sorg, R. V., Fearnley, D. B., and Hart, D. N. J. (1998). Dermal Dendritic Cells Associated with T Lymphocytes in normal Human Skin Display an Activated Phenotype. J. Invest. Dermatol. 111, 841-849. doi:10.1046/ j.1523-1747.1998.00375.x

Mediaty, A., and Neuber, K. (2005). Total and Specific Serum IgE Decreases with Age in Patients with Allergic Rhinitis, Asthma and Insect Allergy but Not in Patients with Atopic Dermatitis. Immun. Ageing 2, 9. doi:10.1186/1742-49332-9

Messingham, K. N., Crowe, T. P., and Fairley, J. A. (2019). The Intersection of IgE Autoantibodies and Eosinophilia in the Pathogenesis of Bullous Pemphigoid. Front. Immunol. 10, 2331. doi:10.3389/fimmu.2019.02331

Miller, L. S., and Modlin, R. L. (2007). Human Keratinocyte Toll-like Receptors Promote Distinct Immune Responses. J. Invest. Dermatol. 127, 262-263. doi:10. 1038/sj.jid.5700559

Nakahara, T., Kido-Nakahara, M., Tsuji, G., and Furue, M. (2021). Basics and Recent Advances in the Pathophysiology of Atopic Dermatitis. J. Dermatol. 48, 130-139. doi:10.1111/1346-8138.15664

Nguyen, Q. P., Deng, T. Z., Witherden, D. A., and Goldrath, A. W. (2019). Origins of CD 4 + Circulating and Tissue-Resident Memory T-Cells. Immunology 157, 3-12. doi:10.1111/imm.13059

Nielsen, M. M., Witherden, D. A., and Havran, W. L. (2017). Г $\delta$ T Cells in Homeostasis and Host Defence of Epithelial Barrier Tissues. Nat. Rev. Immunol. 17, 733-745. doi:10.1038/nri.2017.101

Noe, M. H., Messingham, K., Aust, S., Gross, S., and Fairley, J. A. (2015). Bullous Pemphigoid Patients Exhibit Increased Markers of immunosenescence.2015 Annual Meeting Of the SID. Atlanta, GA, USA. 6-9th May 2015, Meeting Program, S1:LB738.

Otsuka, A., and Kabashima, K. (2015). Mast Cells and Basophils in Cutaneous Immune Responses. Allergy 70, 131-140. doi:10.1111/all.12526

Partridge, L., Fuentealba, M., and Kennedy, B. K. (2020). The Quest to Slow Ageing through Drug Discovery. Nat. Rev. Drug Discov. 19, 513-532. doi:10.1038/ s41573-020-0067-7

Pezzolo, E., and Naldi, L. (2020). Epidemiology of Major Chronic Inflammatory Immune-Related Skin Diseases in 2019. Expert Rev. Clin. Immunol. 16, 155-166. doi:10.1080/1744666X.2020.1719833
Pietkiewicz, P., Gornowicz-Porowska, J., Bowszyc-Dmochowska, M., Bartkiewicz, P., and Dmochowski, M. (2016). Bullous Pemphigoid and Neurodegenerative Diseases: a Study in a Setting of a Central European university Dermatology Department. Aging Clin. Exp. Res. 28, 659-663. doi:10.1007/s40520-015-0459-4

Pilkington, S. M., Barron, M. J., Watson, R. E. B., Griffiths, C. E. M., and BulfonePaus, S. (2019). Aged Human Skin Accumulates Mast Cells with Altered Functionality that Localize to Macrophages and Vasoactive Intestinal Peptide-Positive Nerve Fibres. Br. J. Dermatol. 180, 849-858. doi:10.1111/ bjd. 17268

Pilkington, S. M., Bulfone-Paus, S., Griffiths, C. E. M., and Watson, R. E. B. (2021). Inflammaging and the Skin. J. Invest. Dermatol. 141, 1087-1095. doi:10.1016/j. jid.2020.11.006

Pilkington, S. M., Dearman, R. J., Kimber, I., and Griffiths, C. E. M. (2018a). Langerhans Cells Express Human $\beta$-defensin 3: Relevance for Immunity during Skin Ageing. Br. J. Dermatol. 179, 1170-1171. doi:10.1111/bjd.16770

Pilkington, S. M., Ogden, S., Eaton, L. H., Dearman, R. J., Kimber, I., and Griffiths, C. E. M. (2018b). Lower Levels of Interleukin-1 $\beta$ Gene Expression Are Associated with Impaired Langerhans' Cell Migration in Aged Human Skin. Immunology 153, 60-70. doi:10.1111/imm.12810

Pinti, M., Appay, V., Campisi, J., Frasca, D., Fülöp, T., Sauce, D., et al. (2016). Aging of the Immune System: Focus on Inflammation and Vaccination. Eur. J. Immunol. 46, 2286-2301. doi:10.1002/eji.201546178

Qin, D., Ren, R., Jia, C., Lu, Y., Yang, Q., Chen, L., et al. (2018). Rapamycin Protects Skin Fibroblasts from Ultraviolet B-Induced Photoaging by Suppressing the Production of Reactive Oxygen Species. Cell Physiol Biochem 46, 1849-1860. doi:10.1159/000489369

Rafei-Shamsabadi, D. A., Klose, C. S. N., Halim, T. Y. F., Tanriver, Y., and Jakob, T. (2019). Context Dependent Role of Type 2 Innate Lymphoid Cells in Allergic Skin Inflammation. Front. Immunol. 10, 2591. doi:10.3389/fimmu.2019.02591

Rijken, F., Kiekens, R. C. M., and Bruijnzeel, P. L. B. (2005). Skin-infiltrating Neutrophils Following Exposure to Solar-Simulated Radiation Could Play an Important Role in Photoageing of Human Skin. Br. J. Dermatol. 152, 321-328. doi:10.1111/j.1365-2133.2004.06335.x

Sánchez-Borges, M., Capriles-Hulett, A., Caballero-Fonseca, F., and GonzálezAveledo, L. (2018). Biomarkers of Treatment Efficacy in Patients with Chronic Spontaneous Urticaria. Eur. Ann. Allergy Clin. Immunol. 50, 5-9. doi:10.23822/ EurAnnACI.1764-1489.24

Scharschmidt, T. C., Vasquez, K. S., Pauli, M. L., Leitner, E. G., Chu, K., Truong, H.A., et al. (2017). Commensal Microbes and Hair Follicle Morphogenesis Coordinately Drive Treg Migration into Neonatal Skin. Cell Host \& Microbe 21, 467-477. e5. doi:10.1016/j.chom.2017.03.001

Schwarz, A., Bruhs, A., and Schwarz, T. (2017). The Short-Chain Fatty Acid Sodium Butyrate Functions as a Regulator of the Skin Immune System. J. Invest. Dermatol. 137, 855-864. doi:10.1016/j.jid.2016.11.014

Sperling, T., Ołdak, M., Walch-Rückheim, B., Wickenhauser, C., Doorbar, J., Pfister, H., et al. (2012). Human Papillomavirus Type 8 Interferes with a Novel C/EBP $\beta$-Mediated Mechanism of Keratinocyte CCL20 Chemokine Expression and Langerhans Cell Migration. Plos Pathog. 8, e1002833. doi:10. 1371/journal.ppat.1002833

Suggs, A., Oyetakin-White, P., and Baron, E. (2014). Effect of Botanicals on Inflammation and Skin Aging: Analyzing the Evidence. Inflamm. Allergy-Drug Targets 13, 168-176. doi:10.2174/1871528113666140526163052

Tanei, R., and Hasegawa, Y. (2016). Atopic Dermatitis in Older Adults: A Viewpoint from Geriatric Dermatology. Geriatr. Gerontol. Int. 16 (Suppl. 1), 75-86. doi:10.1111/ggi.12771

Tanei, R., Hasegawa, Y., and Sawabe, M. (2013). Abundant Immunoglobulin E-Positive Cells in Skin Lesions Support an Allergic Etiology of Atopic Dermatitis in the Elderly. J. Eur. Acad. Dermatol. Venereol. 27, 952-960. doi:10.1111/j.1468-3083.2012.04612.x

Thomas, R., Wang, W., and Su, D.-M. (2020). Contributions of Age-Related Thymic Involution to Immunosenescence and Inflammaging. Immun. Ageing 17, 2. doi:10.1186/s12979-020-0173-8

Tsuboi, I., Harada, T., Hirabayashi, Y., Kanno, J., Inoue, T., and Aizawa, S. (2012). Age-related Decline of Mast Cell Regeneration in Senescence-Accelerated Mice (SAMP1) after Chemical Myeloablation Due to Senescent Stromal Cell Impairment. Exp. Biol. Med. (Maywood) 237, 1289-1297. doi:10.1258/ebm. 2012.012158 
Vadasz, Z., and Toubi, E. (2015). The Role of Increased T Helper Cell 2 Cytokine Expression in Skin Weals of Chronic Spontaneous Urticaria: Are They Always Activating Cytokines. Br. J. Dermatol. 172, 1185-1186. doi:10. 1111/bjd.13784

Wasko, R. R., and Horsley, V. (2019). Thin Skinned: Aged Adipocyte Atrophy Impacts Innate Immunity. Trends Immunology 40, 175-177. doi:10.1016/j.it. 2019.01.009

Watanabe, R., Gehad, A., Yang, C., Scott, L. L., Teague, J. E., Schlapbach, C., et al. (2015). Human Skin Is Protected by Four Functionally and Phenotypically Discrete Populations of Resident and Recirculating Memory T Cells. Sci. Transl. Med. 7, 279ra39. doi:10.1126/scitranslmed.3010302

West, H. C., and Bennett, C. L. (2018). Redefining the Role of Langerhans Cells as Immune Regulators within the Skin. Front. Immunol. 8, 1941. doi:10.3389/ fimmu.2017.01941

Williamson, S., Merritt, J., and De Benedetto, A. (2020). Atopic Dermatitis in the Elderly: a Review of Clinical and Pathophysiological Hallmarks. Br. J. Dermatol. 182, 47-54. doi:10.1111/bjd.17896

Wittmann, M., McGonagle, D., and Werfel, T. (2014). Cytokines as Therapeutic Targets in Skin Inflammation. Cytokine Growth Factor. Rev. 25, 443-451. doi:10.1016/j.cytogfr.2014.07.008

Wlaschek, M., Maity, P., Makrantonaki, E., and Scharffetter-Kochanek, K. (2021). Connective Tissue and Fibroblast Senescence in Skin Aging. J. Invest. Dermatol. 141, 985-992. doi:10.1016/j.jid.2020.11.010

Yaar, M., and Gilchrest, B. A. r. A. (1987). Bullous Pemphigoid: Disease of the Aging Immune System. Clin. Dermatol. 5, 135-145. doi:10.1016/0738-081x(87) 90058-7

Yamazaki, S., Nishioka, A., Kasuya, S., Ohkura, N., Hemmi, H., Kaisho, T., et al. (2014). Homeostasis of Thymus-Derived Foxp3+ Regulatory T Cells Is Controlled by Ultraviolet B Exposure in the Skin. J. Immunol. 193, 5488-5497. doi:10.4049/jimmunol.1400985

Ye, L., Mauro, T. M., Dang, E., Wang, G., Hu, L. Z., Yu, C., et al. (2019). Topical Applications of an Emollient Reduce Circulating Pro-inflammatory Cytokine Levels in Chronically Aged Humans: a Pilot Clinical Study. J. Eur. Acad. Dermatol. Venereol. 33, 2197-2201. doi:10.1111/jdv.15540

Zbytek, B., Janjetovic, Z., Tuckey, R. C., Zmijewski, M. A., Sweatman, T. W., Jones, E., et al. (2008). 20-Hydroxyvitamin D3, a Product of Vitamin D3
Hydroxylation by Cytochrome P450scc, Stimulates Keratinocyte Differentiation. J. Invest. Dermatol. 128, 2271-2280. doi:10.1038/jid.2008.62

Zhang, L.-j., Guerrero-Juarez, C. F., Hata, T., Bapat, S. P., Ramos, R., Plikus, M. V., et al. (2015). Dermal Adipocytes Protect against Invasive Staphylococcus aureus Skin Infection. Science 347, 67-71. doi:10.1126/science.1260972

Zhang, L.-j., Chen, S. X., Guerrero-Juarez, C. F., Li, F., Tong, Y., Liang, Y., et al. (2019). Age-Related Loss of Innate Immune Antimicrobial Function of Dermal Fat Is Mediated by Transforming Growth Factor Beta. Immunity 50, 121-136. e5. doi:10.1016/j.immuni.2018.11.003

Zhou, L., Leonard, A., Pavel, A. B., Malik, K., Raja, A., Glickman, J., et al. (2019). Age-specific Changes in the Molecular Phenotype of Patients with ModerateTo-Severe Atopic Dermatitis. J. Allergy Clin. Immunol. 144, 144-156. doi:10. 1016/j.jaci.2019.01.015

Zouboulis, C. C., and Makrantonaki, E. (2011). Clinical Aspects and Molecular Diagnostics of Skin Aging. Clin. Dermatol. 29, 3-14. doi:10.1016/j.clindermatol.2010.07.001

Zuelgaray, E., Boccara, D., Ly Ka So, S., Boismal, F., Mimoun, M., Bagot, M., et al. (2019). Increased Expression of PD1 and CD39 on CD3+ CD4+ Skin T Cells in the Elderly. Exp. Dermatol. 28, 80-82. doi:10.1111/exd.13842

Conflict of Interest: The authors declare that the research was conducted in the absence of any commercial or financial relationships that could be construed as a potential conflict of interest.

Publisher's Note: All claims expressed in this article are solely those of the authors and do not necessarily represent those of their affiliated organizations, or those of the publisher, the editors, and the reviewers. Any product that may be evaluated in this article, or claim that may be made by its manufacturer, is not guaranteed or endorsed by the publisher.

Copyright (c) 2022 Chen, Yang, Song, Zhang and Hao. This is an open-access article distributed under the terms of the Creative Commons Attribution License (CC BY). The use, distribution or reproduction in other forums is permitted, provided the original author(s) and the copyright owner(s) are credited and that the original publication in this journal is cited, in accordance with accepted academic practice. No use, distribution or reproduction is permitted which does not comply with these terms. 